University of Wollongong

Research Online

Faculty of Engineering - Papers (Archive)

Faculty of Engineering and Information

Sciences

2007

\title{
Spatial Resolution of a Small Cubic LYSO Scintillator Crystal Detector with Depth-of-Interaction Capabilities in a Small Animal PET Scanner
}

\author{
L. Perera \\ University of Wollongong
}

M. Lerch

University of Wollongong, mlerch@uow.edu.au

Anatoly B. Rosenfeld

University of Wollongong, anatoly@uow.edu.au

S. R. Meikle

University of Sydney

J. Chan

University of Sydney

Follow this and additional works at: https://ro.uow.edu.au/engpapers

Part of the Engineering Commons

https://ro.uow.edu.au/engpapers/444

\section{Recommended Citation}

Perera, L.; Lerch, M.; Rosenfeld, Anatoly B.; Meikle, S. R.; and Chan, J.: Spatial Resolution of a Small Cubic LYSO Scintillator Crystal Detector with Depth-of-Interaction Capabilities in a Small Animal PET Scanner 2007.

https://ro.uow.edu.au/engpapers/444

Research Online is the open access institutional repository for the University of Wollongong. For further information contact the UOW Library: research-pubs@uow.edu.au 


\section{Spatial Resolution of a Small Cubic LYSO} Scintillator Crystal Detector with Depth-of-Interaction Capabilities in a Small Animal
PET Scanner Lakshal Perera, Student Member, IEEE, Michael Lerch, Member, IEEE, Anatoly Rosenfeld, Senior Member, IEEE Steve R. Meikle, Member, IEEE, and Jan Chan Member, IEEE,

\begin{abstract}
Present Positron Emission Tomography (PET) detectors suffer from degradation of the spatial resolution due to the lack of depth-of-interaction (DOI) information leading to uncertainty in deducing the Lines of Response (LOR) between coincident events. The Centre for Medical Radiation Physics at the University of Wollongong has developed a novel detector module which will provide depth of interaction information while retaining the sensitivity of current scanners. This will result in superior imaging together with the ability to locate smaller lesions. This work focuses on preliminary investigations of the suitability of replacing the bulky scintillator crystals and photomultiplier tubes of traditional PET detector modules with compact $3 \times 3 \times 3 \mathrm{~mm}^{3}$ LYSO scintillator crystals individually coupled to $\mathrm{Si}$ photdetectors.

The simulations were undertaken using GATE, a high level front end for GEANT4, specifically designed for use in the simulation of PET and SPECT. The simulation results quantify the level of improvement that can be potentially gained using a new detector module capable of providing a uniform spatial resolution across the entire field of view (FOV). Data is analysed using a newly developed sinogram binning technique. Simulation results show a resolution of $1.6 \mathrm{~mm}$ FWHM can be obtained for the entire FOV of this scanner (approximately $230 \mathrm{~mm}$ ). This represents an improvement of approximately $75 \%$ in the resolution at the edges of field of view compared to traditional detector module design. Comparison of spatial resolution data for the novel detector module and phoswich detectors is also presented
\end{abstract}

Index Terms-GATE, Small Animal PET, Depth of Interaction

\section{INTRODUCTION}

Most commercial PET scanners use a modular detector design with a block of bismuth germanate (BGO) scintillator crystal coupled to 4 photomultiplier tubes (PMTs) [1]. The light produced from the gamma ray interaction in the crystal is detected by all 4 PMTs and the location of interaction in the axial and tangential directions is determined by the relative response of each of the PMTs using Anger Logic. The spatial resolution of this sytem can be improved by placing small saw

Lakshal Perera, Michael Lerch and Anatoly Rosenfeld are with the Centre for Medical Radiation Physics, University of Wollongong, NSW, 2522 Australia (email: 1p20@uow.edu.au, mlerch@uow.edu.au, anatoly@uow.edu.au) Steve Meikle is with the Brain and Mind Research Institute, University of Sydney, Camperdown, NSW 2050

Jan Chan is with the Biomedical \& Multimedia Information Technology Research Group, School of Information Technologies, University of Sydney, NSW 2006 cut grooves to separate the crystal into many quasi-discrete elements with a typical size of $24 \times 4 \times 4 \mathrm{~mm}^{3}$ [1] (surface faces of crystal are $4 \mathrm{~mm}$ and depth is $24 \mathrm{~mm}$ ). The saw cut grooves ensure light produced in a given element remains in the correct tangential and axial positions when it reaches the photomultiplier tubes. Reducing the size of these crystal elements improves spatial resolution however does not provide any depth of interaction information. The limitations of this approach include

- the fundamental limit on the size of the detector module due to the size of the photomultiplier tubes

- the size of the crystal element due to the thickness of the saw cut

- the amount of dead space in the block increases as crystal elements become smaller which results in reduced detection efficiency when using long thin crystals, offseting the improvement in spatial resolution. [2]

The depth of interaction within the crystal is an important factor which needs consideration when determining the spatial resolution of the system (especially small animal imagers). Most systems cannot determine the exact position within the detector crystal at which the gamma interaction has taken place. As a result, the system determines an abitrary point within the crystal for which it will assume the interaction has taken place (often the centre). For off-centre detector modules, this can result in significant errors in the radial direction as shown in Figure 1. The solid line with arrows indicates the path of 2 unscattered photons. These photons both pass through some scintillator crystal before they interact in another crystal. The system has no method of determining the exact position of interaction within crystal and hence assumes all interactions to take place at a fixed depth in the crystals. The dashed line shows the system determined LOR and there is a noticable difference between the position of this line and the actual LOR. The effect of these radial elongation artefacts increase as the angle between the propogation direction of the photon and the front surface of the detector module moves away from the normal.

Using saw cut grooves in the crystal provides axial and tangential accuracy but it does not allow determination of DOI (an important parameter in small animal PET scanners). 


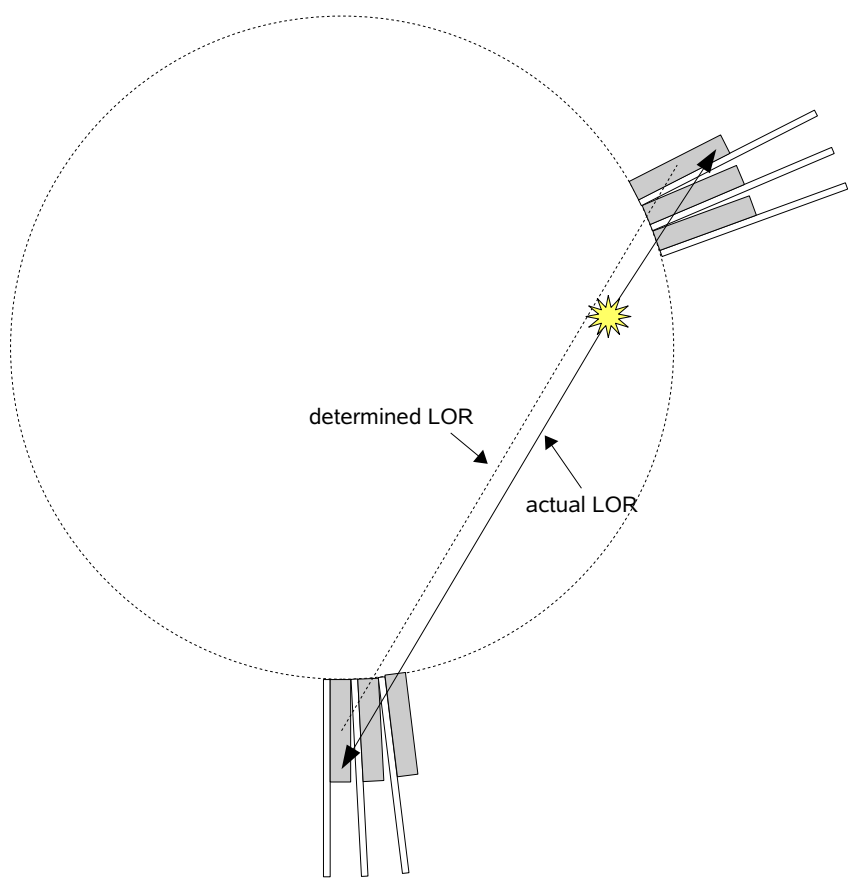

Fig. 1. Depth of Interaction Error

Other techniques which have been used to provide some DOI information include:

- phoswich detectors using mutiple scintillators with unique light decay properties [3] [4] [5]

- dual layer detectors with layers offset by half the crystal pitch [6] [7]

- the ratio of light determined by photodetectors placed at either end of the crystal [8] [9]

- mutiple offset layer detectors using a novel light sharing method [10]

The novel detector module proposed by the CMRP will provide approximately uniform spatial resolution across the entire field of view with the simulations discussed in this paper used to optimise the characteristics of the system.

\section{Novel Detector Module}

The Centre for Medical Radiation Physics (CMRP), University of Wollongong is developing a new (PET) detection module with depth of interaction capability. The new module is simple and robust, minimises module assembly complications and is completely independent of photomultiplier tubes. The new module has also been designed to maximise its flexibility for easy module coupling so as to form a complete, customised, detection module to be used in PET scanners dedicated to human brain and breast, and small animal studies.

The proposed detector module contains an $8 \times 8$ array of optically isolated $3 \times 3 \times 3 \mathrm{~mm}^{3}$ LYSO crystals individually coupled to individual elements of an $8 \times 8 \mathrm{Si}$ photodetector array. The independent coupling of LYSO crystal and photodetector ensures depth of interaction information while maintaining the radial sensitivity of traditional $24 \times 3 \times 3 \mathrm{~mm}^{3}$ used in present PET detectors. The $300 \mu \mathrm{m}$ thick photodetectors

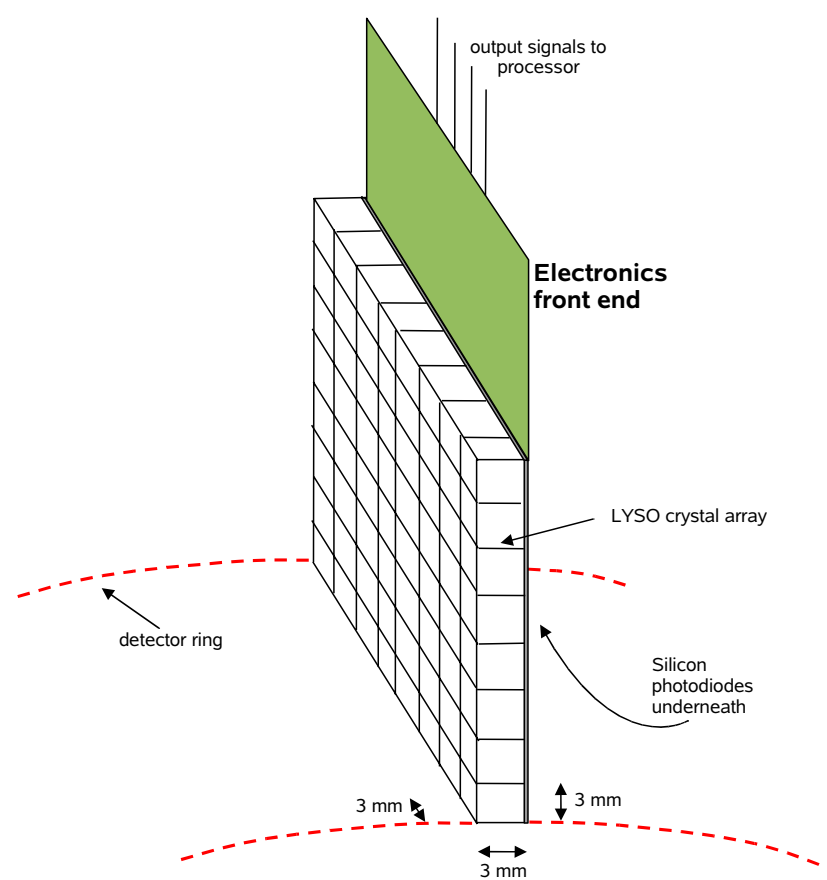

Fig. 2. A schematic of the proposed detector module in "edge on" mode. Each LYSO crystal is individually coupled to a Si photodetector to improve the ability for the system to localise interactions within the scintillator material

are mounted on a $100 \mu \mathrm{m}$ Kapton carrier and a $100 \mu \mathrm{m}$ thick layer of optical paint is placed on the surface of the LYSO modules to prevent light loss resulting in a detector module $3.5 \times 24 \times 24 \mathrm{~mm}^{3}$ in size. This study neglects the thickness of the electronics and investigates the best possible performance of the detector. A schematic of the proposed detector in "edge on" mode is shown in Figure 2.

This work focuses on determining the suitability of the LYSO array a substitute for traditional long crystals using the Monte Carlo package GATE. Comparison of spatial resolutions for each system will be considered.

\section{Monte Carlo Simulation}

GATE (GEANT Application for Tomographic Imaging) is a GEANT4 [11] front end designed specifically for use in PET and SPECT applications [12]. GATE replaces the traditional $\mathrm{C}++$ coding required in GEANT4 with much simpler macro scripting. Individual macro files for various aspects of the simulation define detector geometry, source, phantom, data acquisition, data output and physics processes.

\section{A. Detector Geometry}

The small animal PET scanner modelled in this simulation has an internal diameter of $300 \mathrm{~mm}$ and is $24 \mathrm{~mm}$ wide. As this study is primarily focused on optimisation, only a single ring of the scanner is modeled and it is anticipated the final prototype will be constructed using numerous sets of rings, providing a much larger field of view.

Eight layers of $3 \times 3 \times 3 \mathrm{~mm}^{3}$ LYSO crystals are placed on the detector ring in a row of 8 crystals aligned along the 


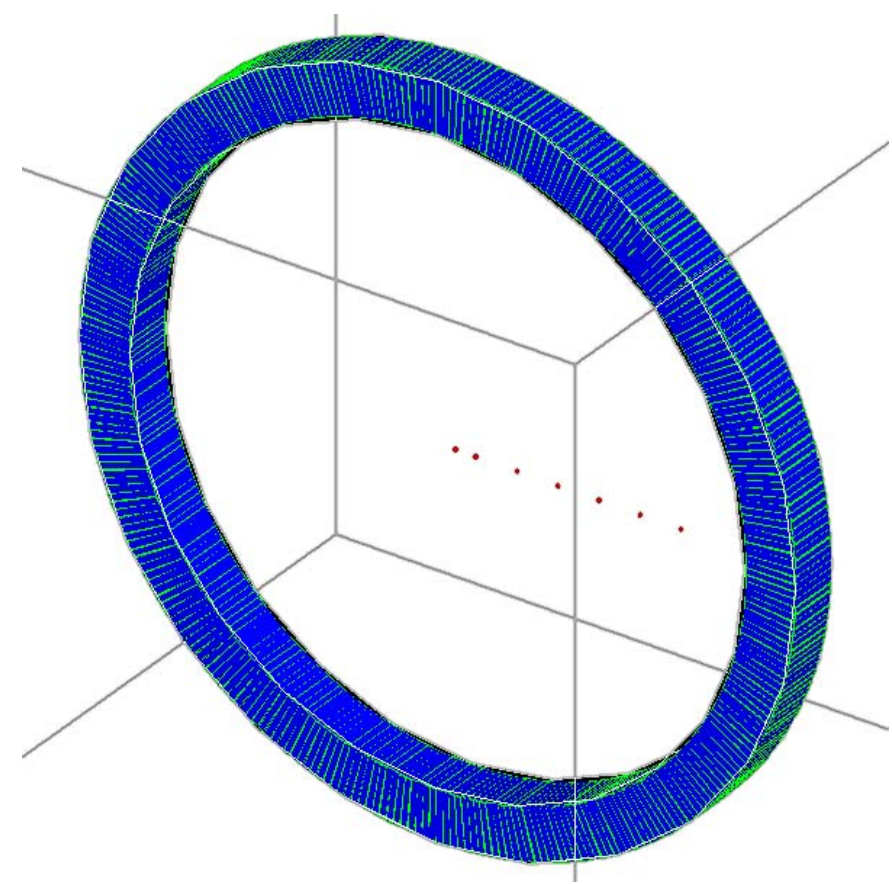

Fig. 3. A typical simulation geometry showing a scanner with an $8 \times 8$ array of $3 \times 3 \times 3 \mathrm{~mm}^{3}$ crystals and 7 point sources placed at various points along the $x$-axis in the field of view.

axis of the scanner, creating an array of 64 crystals per detector module as seen in Figure 2. A total of 314 modules were placed around the detector ring and figure of the simulation is shown in Figure 3.

\section{B. Sources and Phantoms}

Two separate studies using a simple water phantom and no phantom are considered. The "world volume" used in both sets of simulation consists of air. The water phantom used in the simulations is cylindrical with a radius of $120 \mathrm{~mm}$ and a length of $100 \mathrm{~mm}$. The axis of the cylinder is placed parallel to the axis of the detector and the centre of the phantom was placed at the centre of field of view (FOV). The length of the phantom is significantly larger than the scanner width $(24 \mathrm{~mm})$ to ensure a large volume for potentially scattered photons to emerge from.

A $1 \mathrm{MBq}$ point source (with radius of $0.0001 \mathrm{~mm}$ ) is placed at the centre of FOV and at points 10, 30, 50, 70, 90, $110 \mathrm{~mm}$ across the FOV. The spectrum of the sources is selected in GATE to model that of Flourine-18.

In order to reduce the computational time involved, the simulations without the water phantom has the source emitting a fan beam towards the direction of the detectors. As the"world" volume consisted of only air, few photons are expected to scatter from this volume into the detectors suggesting this approximation was suitable. Preliminary studies showed very few photons scattering from the water phantom into the detector therefore the water phantom was given a background activity of $10000 \mathrm{MBq}$.

"Standard" GEANT4 processes were selected for the photoelectic effect and Compton scattering ensuring these interactions were modelled down to $10 \mathrm{keV}$. The Low Energy
Processes allow interactions down to $250 \mathrm{eV}$ to be modelled, however this level of accuracy in unrequired in this study. Electrons were not transported as doing so would increase the simulation time greatly.

\section{Data Acquisition}

The lower and upper level discriminator values are set to $150 \mathrm{keV}$ and $650 \mathrm{keV}$ respectively. Offline processing allows only coincidences within the specified energy range to be read in from the output data and processed. The coincidence window is $10 \mathrm{~ns}$ with coincidences occurring in modules separated by less than 3 other modules being ignored.

\section{Simulation Hardware}

The simulations were undertaken on the CMRP High Performance Computing Grid, consisting of $283 \mathrm{GHz}$ processors. As a parallel processing version of GATE was unavailable at the time of simulation, multiple simulations were run with independent random seeds and data collated for analysis.

\section{E. Data Analysis}

The data output of the simulations is ASCII data containing the cartesian co-ordinates, the energy deposited in the crystal and the dectector IDs (as defined according to known conventions in GATE) of each crystal containing a photon deposting energy above threshold from the coincidence. The data is analysed using a newly developed sinogram rebinning algorithm. Sinograms have been extensively used to store information from single layered detector systems, however a paradigm for storing information from multiple layers of crystal in a single sinogram has to our knowledge not been developed.

The method devloped for producing the sinogram from the multiple layers of data is specifically designed for the data output of these Monte Carlo studies. It accepts the positions of the 2 photons in the interaction and determines the angle of this LOR with respect to the system and the radial distance from the origin to this LOR. The pixel value of the sinogram element representing the determined angle and radial distance is then incremented.

The midpoint of the LOR along the axis of each detector ring is also determined using the z-coordinates of the two interactions as shown in Figure 4. As the width of each detector module is small $(3 \mathrm{~mm})$, coincidences are allowed to occur between the two edgemost crystals in that ring however when a final prototype is developed, coincidences will only be allowed between independent detector rings according to traditional ring span values. Eight sinograms are produced for each detector ring, each representing the LORs originating from the 8 axial crystals within that detector. Each of these slices are used to reconstruct indepentent images which can be overlayed to produce a 3D image.

Placing interactions from multiple layers of crystal into a single sinogram effectively results in upsampling along the projection angle axis of the sinogram. That is, smaller angular increments are possible in the projection angle as 


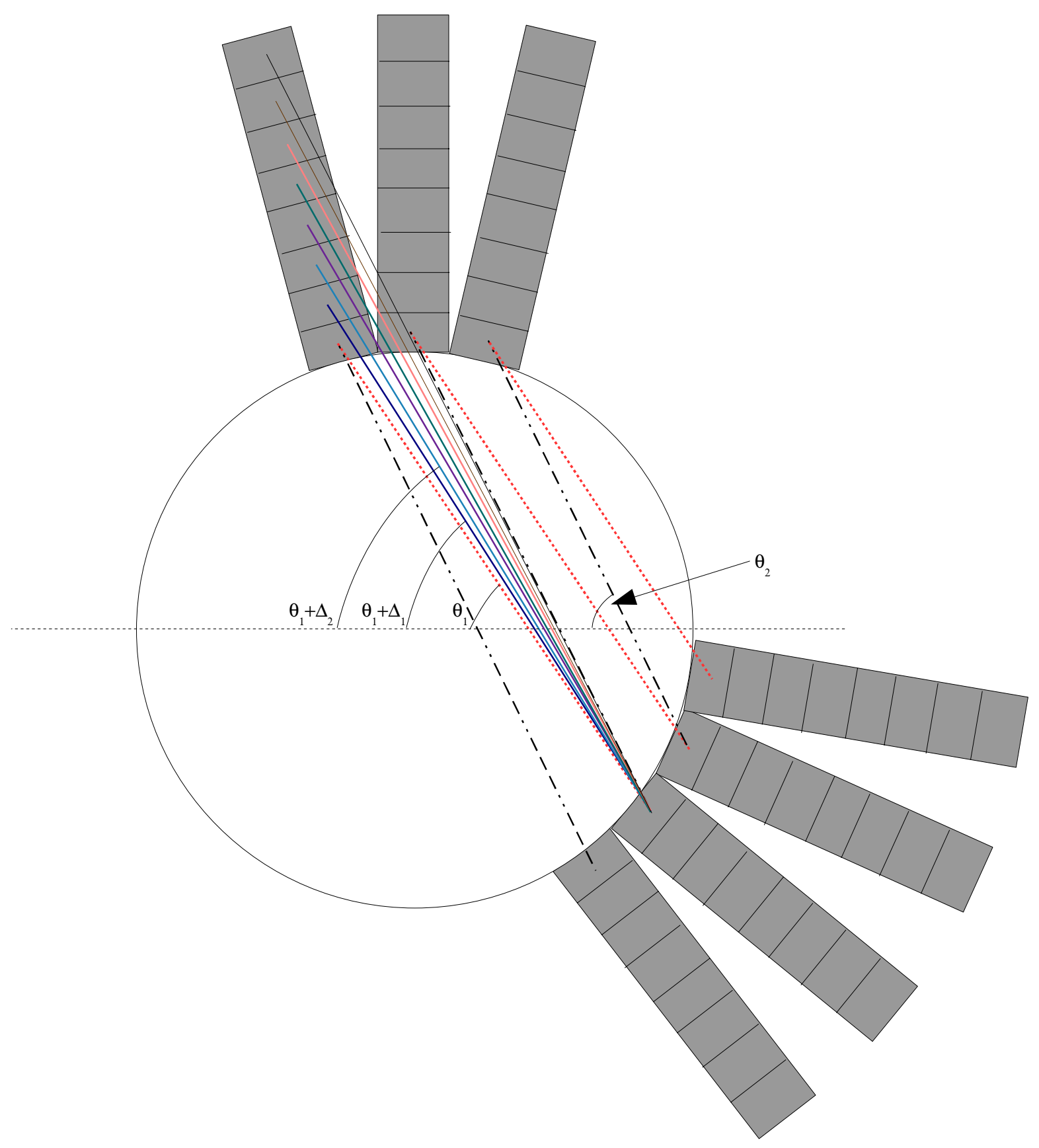

Fig. 5. Multiple layers of data can allow smaller increments in the projection angle resulting in improved resolution for off centre sources (not to scale)

shown in Figure 5. The angles of projection described by the lines at angles $\phi_{1}$ and $\phi_{2}$ are adjacent angles of projection in a traditional scanner design. Depth of interaction allows projection angles at values inbetween the existing projection angles expressed by lines at angles of $\phi_{1}+\Delta_{1}$ and $\phi_{1}+\Delta_{2}$ and all other unlabled lines between the existing projection angles $\phi_{1}$ and $\phi_{2}$. Additional projection angles result in an increased number of pixels in the constructed sinogram, hence increased resolution. However, the improvement is negligible at the centre of FOV as depth of interaction provides no further information than available from a single layer of crystal.

Having 8 layers of crystal does not necessarily provide an 8 times upsampling in this axis. This is due to certain pro- jection angles resulting from LORs from deeper level crystals having projection angles similar or identical to LOR's already commencing from adjacent detector modules. Subsequently a conservative upsampling of two times has been considered for this study. The height and width of the sinogram are dependent on the number of opposite detector pairs present. As the $3 \times 3 \times 3 \mathrm{~mm}^{3}$ crystal simulations have 314 detector modules, 157 detector pairs were present. As two times upsampling has been implemented in the sinogram binning stage, a final sinogram of $314 \times 314$ pixels is produced.

The images were reconstructed using an OSEM reconstruction algorithm written in IDL[13] by Dr Meikle from the Brain and Mind Research Institute at The University of Sydney. 


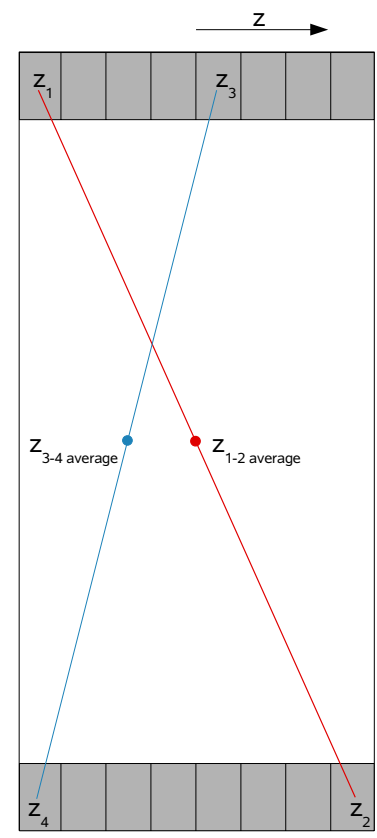

Fig. 4. Cross section view of detector ring showing determination of z-axis position within each individual detector ring (not to scale)

\section{RESULTS}

Two line profile of reconstructions of each point sources position for $24 \times 3 \times 3 \mathrm{~mm}^{3}$ and $3 \times 3 \times 3 \mathrm{~mm}^{3}$ crystals are shown in Figure 6(a) and 6(b) with the reconstructed images shown in $6(\mathrm{c})$ and $6(\mathrm{~d})$ respectively.. The spatial resolution across the FOV is shown in Figure 7.

The resolution of the source remains approximately constant at $1.6 \mathrm{~mm}$ FWHM across the entire field of view when using 8 layers of $3 \times 3 \times 3 \mathrm{~mm} 3$ crystals. When using a single $24 \times 3 \times 3 \mathrm{~mm}^{3}$ crystal in order to retain the same sensitivity, the radial elongation artifact is clearly observable with degrading image quality as the sources are moved further off centre. The $3 \times 3 \times 3 \mathrm{~mm} 3$ crystals provide a $75 \%$ improvement in spatial resolution at the edge of FOV (ie. $110 \mathrm{~mm}$ from the centre of FOV). The number of detected events for each source position remains constant, however as these counts are spread out over a larger area, the brightness of the artifact affected sources decreases.

Smaller crystals will provide more DOI information and hence improve the spatial resolution. These simulations allow the exact cartesian coordinates of the interaction position to be recorded. Using these cartesian coordinates to bin the sinogram, the FWHM of the reconstructed sources across the entire FOV is shown in Figure 8. This reconstruction failed to resolve the $0.0001 \mathrm{~mm}$ sources. This is obviously due to the selected sinogram dimensions ( 314 x 314 pixels as there are 157 detector pairs and an upsampling of 2 times has been considered) which theoretically limits the resolution. One can however use this to make a comparison with the data obtained with the $3 \times 3 \times 3 \mathrm{~mm}^{3}$ crystals. Increasing the dimensions of the sinogram (by increasing the number of detector modules) will improve the performance of the

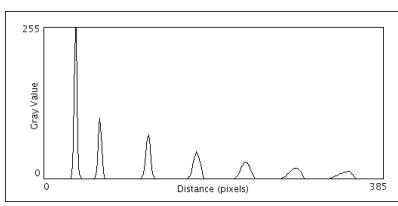

(a)

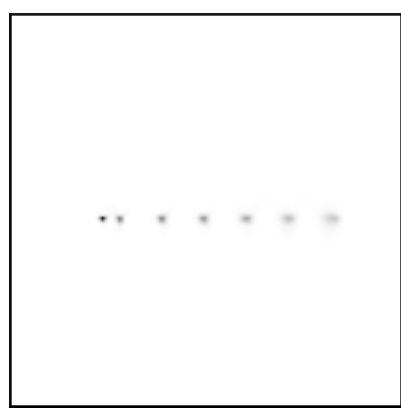

(c)

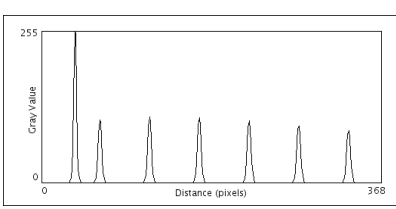

(b)

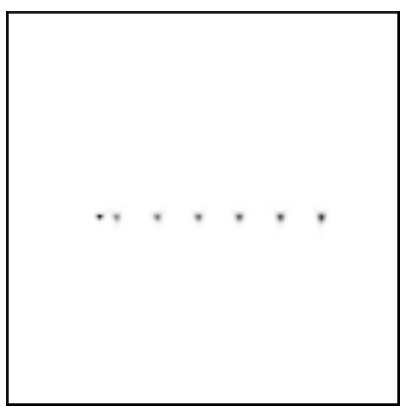

(d)
Fig. 6. 6(a) and 6(b) are the line profiles of the reconstructed point sources across the FOV. Figure 6(c) shows the reconstructed image for a system using $24 \times 3 \times 3 \mathrm{~mm}^{3}$ crystals and Figure $6(\mathrm{~d})$ for $3 \times 3 \times 3 \mathrm{~mm}^{3}$ crystals

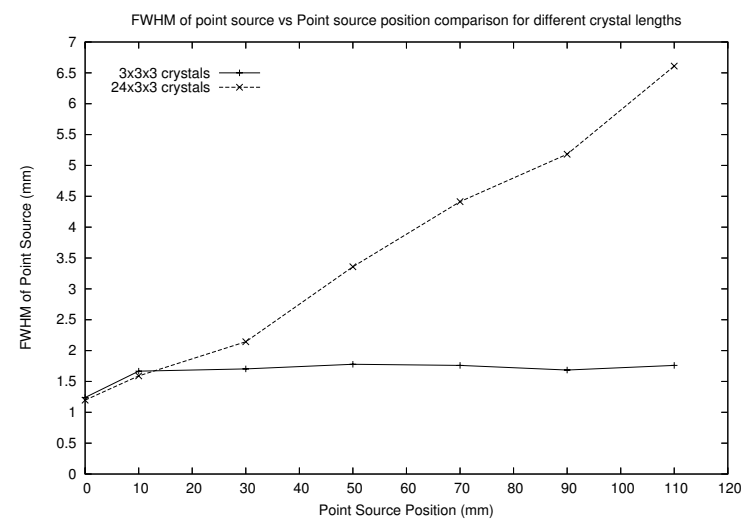

Fig. 7. Spatial resolution for $3 \times 3 \times 3 \mathrm{~mm}^{3}$ and $24 \times 3 \times 3 \mathrm{~mm}^{3}$ crystals

cartesian coordinate sinogram binning technique, however factors such as small angle Compton Scattering, positron range and energy resolution of the crystals provide a finite limit on the capabilities of the detectors. Furthermore, decreasing the crystal size will cause a degredation in the count statistics for a given pixel and considerations need to be made due to increased electronic complexity.

For the $314 \times 314$ pixel image generated, each pixel in the image represents approximately $1.1 \mathrm{~mm}$ of real space, causing the theoretical limit of resolution to approach this value.

In order to further optimise the geometric configuration of the detector module, studies were undertaken to determine the optimal length of the crystal. It is desirable to have longer crystals as sensetivity is improved, however depth of interaction error becomes significant. Multiple layers of crystal is suggest by this study allow a compromise between a larger effective scintillator length and sensitivity considerations must however be made as reducing the number of layers of crystal 


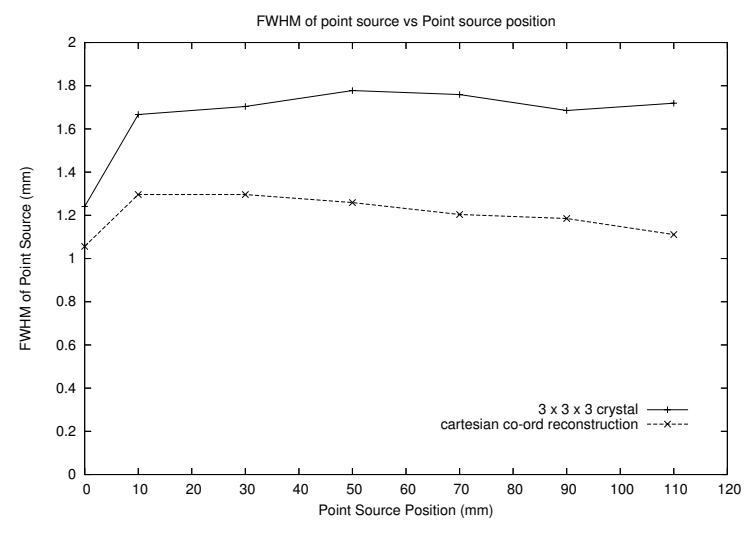

Fig. 8. Spatial resolutions for $3 \times 3 \times 3 \mathrm{~mm}^{3}$ crystals and cartesian coordinate reconstruction

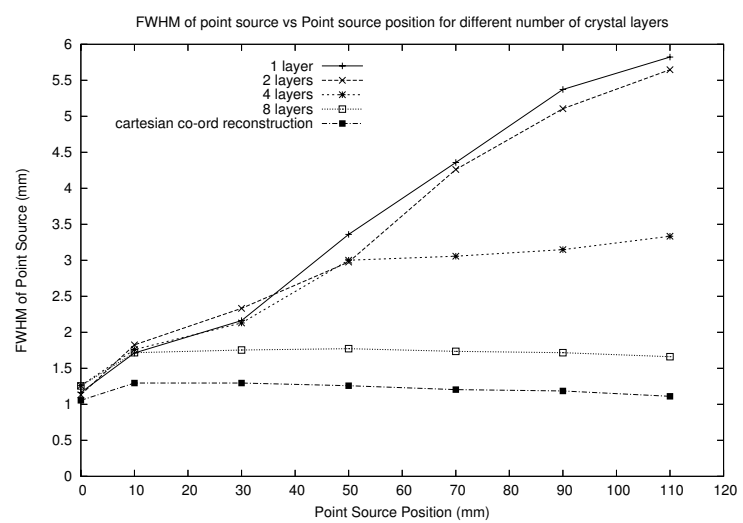

Fig. 9. Spatial resolutions for different numbers of depth of interaction information layers

results in a decrease in the required number of channels from each detector module, making the design, manufacture and operation of the electronic backend less complicated.

To observe the spatial resolution of the system as the crystal length increases, the sinogram binning software was given the capability to group crystals together, effectively rebinning coincidences into radially longer crystals while retaining the same sensitivity in the overall scanner design. That is, if a study with 4 radial layers (effectively $6 \times 3 \times 3 \mathrm{~mm}^{3}$ crystals) was to be compared with the 8 layer studies undertaken, the events from every 2 layers are grouped and the LOR is created from the boundary of the two $3 \times 3 \times 3 \mathrm{~mm}^{3}$ crystals. Similar rebinning processes can be carried out to model 2 layers and single layer detector geometries.

The results for the spatial resolution obtained for $8,4,2$ layers and 1 layer are shown in Figure 9. Resolutions for the cartesian coordinate rebinning are also shown to display the theoretically optimal performance.

The results of this study indicate that using less than 8 layers compromise the spatial resolution at the expense of decreased electronic complexity. The results also indicate the 8 layers of crystals provide superior performance to a 2 layer or 4 layer phoswich devices with smaller ring diameters [3][14][15][16].

\section{CONCLuSion}

This study has shown the proposed detector geometry using $3 \times 3 \times 3 \mathrm{~mm}^{3}$ voxels provides excellent resolution of approximately $1.6 \mathrm{~mm}$ FWHM which remains constant for the entire FOV. The proposed system outperforms current single layer and phoswich systems while retaining similar sensitivity. Further studies investigating optimal crystal size and detector energy windows will be undertaken.

\section{REFERENCES}

[1] M.E. Casey and R. Nutt. A Multicrystal Two Dimensional BGO Detector System. IEEE Trans. Nuc. Sci., page 460, 1986.

[2] S.R. Cherry, Y. Shao, M.P. Tornai, S. Siegel, A.R. Ricci, and M.E. Phelps. Collection of Scintillation Light from Small BGO Crystals. IEEE Trans. Nuc. Sci., 42(3):1058, 1995.

[3] M. Dahlbom, L.R. MacDonals, L. Eriksson, M. Paulus, M. Andreaco, M.E. Casey, and C. Moyers. Performance of a YSO/LSO Phoswitch Detector for use in a PET/SPECT System. IEEE Trans. Nuc. Sci., 44:1114, 1998.

[4] J. Seidel, J. Vaquero, S. Siegel, W. R. Gandler, and M. V. Green. Depth Identication Accuracy of a Three-Layer Phoswich PET Detector Module. IEEE Trans. Nuc. Sci., 46:485, 1999.

[5] U. Heinrichs, U. Pietrzyk, and K. Ziomons. Design Optimization of the PMT-ClearPET Prototypes Based on Simulation Studes with GEANT3. IEEE Trans. Nuc. Sci., 50:1428, 2003.

[6] H. Liu, T. Omura, M. Watanabe, and T. Yamashita. Development of a Depth of Interaction Detector for Gamma-Rays. page 182, 2001.

[7] N. Zhang, C. J. Thompson, D. Togane, F. Cayouette, K. Q. Nguyen, and M. L. Camborde. Anode Position and Last Dynode Timing Circuits for Dual-Layer BGO Scintillator with PS-PMT Based Modular PET Detectors. IEEE Trans. Nuc. Sci., 49:2203, 2002.

[8] W. W. Moses and S. E. Derenzo. Design Studies for a PET Detector Module using a PIN Photodiode to Measure Depth of Interaction. IEEE Trans. Nuc. Sci., 41:1441, 1994.

[9] J. S. Huber, W. W. Moses, M. S. Andreaco, and O. Petterson. An LSO Scintillator Array for a PET Detector Module with Depth of Interaction Measurement. IEEE Trans. Nuc. Sci., 48:684, 2001.

[10] H. Murayama, H. Ishibashi, H. Uchida, T. Omura, and T. Yamashita. Depth Encoding Multicrystal Detectors for PET. IEEE Trans. Nuc. Sci., 45:1152, 1998.

[11] S. Agostinelli et al. Geant4-a simulation toolkit. Nucl Instrum Meth A, 506(3):250, 2003.

[12] S Jan et al. GATE: a simulation toolkit for PET and SPECT. Phys. Med. Biol., 49:4543, 2004.

[13] http://www.ittvis.com/idl/.

[14] J. Seidel, J. Vaquero, and M. V. Green. Resolution Uniformity and Sensitivity of the NIH ATLAS Small Animal PET Scanner:Comparison to Simulated LSO Scanners Without Depth-of-Interaction Capability. IEEE Trans. Nuc. Sci., 50(5):1347, 2003.

[15] Y.H. Chung, Y. Choi, G. Cho, Y.S. Choe, K.H. Lee, and B.T. Kim Optimising of Dual Layer Phoswitch Detector Consisting of LSO and LuYAP for Small Animal PET. IEEE Trans. Nuc. Sci., 52(1):217, 2005.

[16] J.-B. Mosset, O. Devroede, M. Krieguer, M. Rey, J.-M. Vieira, J. H. Jung, C. Kuntne, M. Streun, K. Ziemons, E. Auffray, P. Sempere-Roldan, P. Lecoq, P. Bruyndonckx, J.-F. Loude, S. Tavernier, and C. Morel. Development of an Optimised LSO/LuYAP Phoswich Detector Head for the Lausanne ClearPET Demonstrator. IEEE Trans. Nuc. Sci., 53(1):25, 2006. 